\title{
SEVERAL ASPECTS OF APPLYING DISTRIBUTIONS TO ANALYSIS OF GRAVITATIONAL SHOCK WAVES IN GENERAL RELATIVITY
}

\author{
I. YA. AREF'EVA, A. A. BAGROV, AND L. V. JOUKOVSKAYA
}

Dedicated to Ludwig Dmitrievich Faddeev on the occasion of his 75 th birthday

\begin{abstract}
The ultrarelativistic limit of the stationary Schwarzschild solution in de Sitter space-time of dimension $D=4,5$ is considered. A regularization procedure required for the mathematically correct definition of such a limit is formulated. Some auxiliary statements are proved.
\end{abstract}

\section{§1. INTRODUCTION}

Shock waves describe the ultrarelativistic limit of boosted stationary solutions. The study of shock waves is an important and actively developing field of research within general relativity. Mathematically, this limiting procedure is realized by an application of the boost to the metric form $d s^{2}=g_{i k}(x) d x^{i} d x^{k}(i, k=0, \ldots, D-1)$. The boost corresponds to the change of the coordinate system such that in the new system the source of the gravitational field moves with velocity $v$ in some given direction, under the assumption that the limit passage $v \rightarrow 1$ is performed, which corresponds to a source moving near the speed of light, and that the source energy is finite, while the mass of the source goes to zero. For example, the boost along the coordinate $x^{1}$ can be realized by the change of coordinates

$$
\begin{aligned}
\widetilde{x}^{0} & =\gamma\left(x^{0}+v x^{1}\right), \quad \gamma \equiv\left(1-v^{2}\right)^{-1 / 2}, \\
\widetilde{x}^{1} & =\gamma\left(v x^{0}+x^{1}\right), \\
\widetilde{x}^{m} & =x^{m}, \quad m=2, \ldots, D-1,
\end{aligned}
$$

followed by the limit passage

$$
d \widetilde{s}^{2}=\lim _{v \rightarrow 1} d s^{2} .
$$

A physical consequence of this change of the metric is the Lorenz contraction of the gravitational field in the direction of the moving particle. In the limit case the gravitational field is located on the hypersurface $x^{0}+x^{1}=0$, transversal to the velocity vector of the gravitational source.

For the first time, the procedure was realized in the paper [1] for the simplest case of the initial Schwarzschild metric. Since then, several solutions of this type have been found and analyzed; see, e.g., 2]-9.

The recent interest in shock waves is related to the TeV-gravity scenario [10]- 13]. According to this scenario, the gravitational interaction of elementary particles may

2010 Mathematics Subject Classification. Primary 83C35, 83C75.

Key words and phrases. dS space-time, gravitational waves, regularization.

This paper was partially supported by RFBR grant 09-01-12179-ofi-m and the State contract of Federal agency on science and technology N 02.740.11.5057. I.A. was also supported in part by the NS grant NS-795.2008.1. 
be observed in experiments at large colliders [14]-[17]. There is also a conjecture that shock waves may serve as good models for describing gravitational fields of relativistic elementary particles [18]-23].

It should be noted that, in theories with such space-time, several mathematical problems require special consideration. First, the limit (2) is not well defined for all initial metrics. Second, in the cases where this limit can be taken, the resulting gravitational field is located on a surface whose dimension is less than that of the initial space-time, which means that the support of some metric elements $g_{i k}$ can have zero measure. Instead of a smooth tensor field we get a distribution.

A possibility for dealing with gravitational fields whose metric components are distributions was studied in 24-27. In the present paper, we consider the question of the existence of the limit (2). We propose examples of metrics for which such a limit cannot be realized in a naive way, and we construct a regularization procedure that makes it possible to extend the class of spaces for which the Hilbert-Einstein equations admit gravitational shock wave solutions.

\section{§2. Regularization of the Limit PRocedure}

To pass to the coordinate system in which the gravitational field source moves with the speed of light, we need to find the following limit:

$$
\lim _{v \rightarrow 1} \gamma f\left(\gamma^{2}\left(x^{0}+v x^{1}\right)^{2}\right), \quad \gamma \equiv\left(1-v^{2}\right)^{-1 / 2} .
$$

The following statement is proved easily.

Lemma 1. For any integrable function $f(x) \in \mathcal{L}^{1}(\mathbb{R})$, the following identity is true in the sense of distributions on the space of continuous functions $\mathcal{C}^{0}(\mathbb{R})$ :

$$
\lim _{v \rightarrow 1} \gamma f\left(\gamma^{2}\left(x^{0}+v x^{1}\right)^{2}\right)=\delta\left(x^{0}+x^{1}\right) \int_{-\infty}^{\infty} f\left(x^{2}\right) d x
$$

Proof. Consider the integral

$$
\gamma \int_{-\infty}^{\infty} f\left(\gamma^{2}\left(x^{0}+v x^{1}\right)^{2}\right) g\left(x^{0}\right) d x^{0}
$$

where $g(x)$ is a continuous function. Denote

$$
\gamma\left(x^{0}+v x^{1}\right)=x .
$$

The integral (3) takes the form

$$
\int_{-\infty}^{\infty} f\left(x^{2}\right) g\left(-v x^{1}+\frac{1}{\gamma} x\right) d x
$$

and the continuity of $g(x)$ allows us to write

$$
\lim _{v \rightarrow 1} \int_{-\infty}^{\infty} f\left(x^{2}\right) g\left(-v x^{1}+\frac{1}{\gamma} x\right) d x=g\left(-x^{1}\right) \int_{-\infty}^{\infty} f\left(x^{2}\right) d x,
$$

which proves Lemma 1.

This simple result is well known and was presented, e.g., in [2]. However, it is valid only if

$$
\int_{-\infty}^{\infty} f\left(x^{2}\right) d x<\infty
$$

This integrability condition fails if the source is embedded in a space-time that possesses the event horizon. This concerns, for example, the case of the de Sitter space-time. 
Below it will be shown that, to obtain a shock wave propagating in the dS space-time, one must give a meaning to the following limit procedure in the sense of distributions in the argument $Y$ :

$$
\lim _{\gamma \rightarrow \infty}\left(\frac{\gamma}{\left(Z^{2}-\gamma^{2} Y^{2}\right)^{2}} f\left(\gamma^{2} Y^{2}\right)\right)
$$

It is well known that expressions of the form $\frac{1}{Z^{2}-\gamma^{2} Y^{2}}$, as well as of the form $\frac{1}{\left(Z^{2}-\gamma^{2} Y^{2}\right)^{2}}$, do not determine distributions in $Y$ for any $\gamma \neq 0$ [28, 29]. Therefore, to get a mathematically correct result, we need a regularization procedure.

Lemma 2. The identity

$$
\lim _{\gamma \rightarrow \infty}\left(\frac{\gamma}{Z^{2}-\gamma^{2} Y^{2}}\right)_{\text {reg }} f\left(\gamma^{2} Y^{2}\right)=\delta(Y) \int_{-\infty}^{\infty}\left(\frac{1}{Z^{2}-x^{2}}\right)_{\text {reg }} f\left(x^{2}\right) d x
$$

is true in the sense of distributions on the space $\mathcal{C}^{0}(\mathbb{R})$. Here regularization is understood as follows:

$$
\begin{aligned}
\int_{-\infty}^{\infty} & \left(\frac{\gamma}{Z^{2}-\gamma^{2} Y^{2}}\right)_{\mathrm{reg}} f\left(\gamma^{2} Y^{2}\right) g(Y) d Y \\
= & \int_{|Z-x|<\epsilon} \frac{1}{2 Z} \frac{f\left(x^{2}\right) g\left(\frac{x}{\gamma}\right)-f\left(Z^{2}\right) g\left(\frac{Z}{\gamma}\right)}{Z-x} d x+\int_{|Z-x|>\epsilon} \frac{1}{2 Z} \frac{f\left(x^{2}\right) g\left(\frac{x}{\gamma}\right)}{Z-x} d x \\
& +\int_{|Z+x|<\epsilon} \frac{1}{2 Z} \frac{f\left(x^{2}\right) g\left(\frac{x}{\gamma}\right)-f\left(Z^{2}\right) g\left(-\frac{Z}{\gamma}\right)}{Z+x} d x+\int_{|Z+x|>\epsilon} \frac{1}{2 Z} \frac{f\left(x^{2}\right) g\left(\frac{x}{\gamma}\right)}{Z+x} d x
\end{aligned}
$$

Remark. When defining the regularization (4), we assume that:

- we have

$$
\int_{-\infty}^{\infty}\left(\frac{\gamma}{Z^{2}-\gamma^{2} Y^{2}}\right)_{\mathrm{reg}} f\left(\gamma^{2} Y^{2}\right) g(Y) d Y \equiv \int\left(\frac{1}{Z^{2}-x^{2}}\right)_{\mathrm{reg}} f\left(x^{2}\right) g\left(\frac{x}{\gamma}\right) d x
$$

- the regularization of the right-hand side of (5) is defined by

$$
\int_{-\infty}^{\infty}\left(\frac{1}{Z^{2}-x^{2}}\right)_{\mathrm{reg}} f\left(x^{2}\right) d x=\left(\int \frac{f\left(x^{2}\right)}{2 Z}\left(\frac{1}{Z-x}\right)_{\mathrm{reg}} d x+\int \frac{f\left(x^{2}\right)}{2 Z}\left(\frac{1}{Z+x}\right)_{\mathrm{reg}} d x\right) ;
$$

- the two terms on the right in (6) are regularized naturally:

$$
\begin{aligned}
& \int_{-\infty}^{\infty}\left(\frac{1}{Z-x}\right)_{\mathrm{reg}} f\left(x^{2}\right) d x=\left(\int_{|Z-x|<\epsilon} \frac{f\left(x^{2}\right)-f\left(Z^{2}\right)}{Z-x} d x+\int_{|Z-x|>\epsilon} \frac{f\left(x^{2}\right)}{Z-x} d x\right), \\
& \int_{-\infty}^{\infty}\left(\frac{1}{Z+x}\right)_{\mathrm{reg}} f\left(x^{2}\right) d x=\left(\int_{|Z+x|<\epsilon} \frac{f\left(x^{2}\right)-f\left(Z^{2}\right)}{Z+x} d x+\int_{|Z+x|>\epsilon} \frac{f\left(x^{2}\right)}{Z+x} d x\right) .
\end{aligned}
$$


Proof. Lemma 2 is proved by direct calculation of the limit as $\gamma \rightarrow \infty$. We use the continuity of the function $g(x)$. On the right-hand side of (4) we obtain

$$
\begin{aligned}
\lim _{\gamma \rightarrow \infty}( & \left.\left(\frac{\gamma}{Z^{2}-\gamma^{2} Y^{2}}\right)_{\mathrm{reg}} f\left(\gamma^{2} Y^{2}\right), g\right) \\
= & \lim _{\gamma \rightarrow \infty}\left(\int_{|Z-x|<\epsilon} \frac{1}{2 Z} \frac{f\left(x^{2}\right) g\left(\frac{x}{\gamma}\right)-f\left(Z^{2}\right) g\left(\frac{Z}{\gamma}\right)}{Z-x} d x+\int_{|Z-x|>\epsilon} \frac{1}{2 Z} \frac{f\left(x^{2}\right) g\left(\frac{x}{\gamma}\right)}{Z-x} d x\right) \\
& +\lim _{\gamma \rightarrow \infty}\left(\int_{|Z+x|<\epsilon} \frac{1}{2 Z} \frac{f\left(x^{2}\right) g\left(\frac{x}{\gamma}\right)-f\left(Z^{2}\right) g\left(-\frac{Z}{\gamma}\right)}{Z+x} d x+\int_{|Z+x|>\epsilon} \frac{1}{2 Z} \frac{f\left(x^{2}\right) g\left(\frac{x}{\gamma}\right)}{Z+x} d x\right) \\
= & \int_{|Z-x|<\epsilon} \frac{1}{2 Z} \frac{f\left(x^{2}\right) g(0)-f\left(Z^{2}\right) g(0)}{Z-x} d x+\int_{|Z-x|>\epsilon} \frac{1}{2 Z} \frac{f\left(x^{2}\right) g(0)}{Z-x} d x \\
& +\int_{|Z+x|<\epsilon} \frac{1}{2 Z} \frac{f\left(x^{2}\right) g\left(\frac{x}{\gamma}\right)-f\left(Z^{2}\right) g(0)}{Z+x} d x+\int_{|Z+x|>\epsilon} \frac{1}{2 Z} \frac{f\left(x^{2}\right) g(0)}{Z+x} d x \\
= & g(0)\left(\int_{|Z-x|<\epsilon} \frac{1}{2 Z} \frac{f\left(x^{2}\right)-f\left(Z^{2}\right)}{Z-x} d x+\int_{|Z-x|>\epsilon} \frac{1}{2 Z} \frac{f\left(x^{2}\right)}{Z-x} d x\right) \\
& +g(0)\left(\int_{|Z+x|<\epsilon} \frac{1}{2 Z} \frac{f\left(x^{2}\right)-f\left(Z^{2}\right)}{Z+x} d x+\int_{|Z+x|>\epsilon} \frac{1}{2 Z} \frac{f\left(x^{2}\right)}{Z+x} d x\right) \\
= & g(0)\left(\int \frac{f\left(x^{2}\right)}{2 Z}\left(\frac{1}{Z-x}\right)_{\mathrm{reg}} d x+\int \frac{f\left(x^{2}\right)}{2 Z}\left(\frac{1}{Z+x}\right)_{\mathrm{reg}} d x\right) \\
\equiv & g(0) \int f\left(x^{2}\right)\left(\frac{1}{Z^{2}-x^{2}}\right)_{\mathrm{reg}} d x .
\end{aligned}
$$

Using this auxiliary result, we can prove a similar relation for functions with higher singularity.

Lemma 3. The identity

$$
\lim _{\gamma \rightarrow \infty}\left(\frac{\gamma}{\left(Z^{2}-\gamma^{2} Y^{2}\right)^{2}}\right)_{\text {reg }} f\left(\gamma^{2} Y^{2}\right)=\delta(Y) \int\left(\frac{1}{\left(Z^{2}-x^{2}\right)^{2}}\right)_{\text {reg }} f\left(x^{2}\right) d x
$$

is true in the sense of distributions on the space $\mathcal{C}^{0}(\mathbb{R})$. Here we apply the regularization (7)

$$
\begin{aligned}
& \left(\left(\frac{\gamma}{\left(Z^{2}-\gamma^{2} Y^{2}\right)^{2}}\right)_{\mathrm{reg}} f\left(\gamma^{2} Y^{2}\right), g\right) \\
& \quad=\int_{|Z-x|<\epsilon} \frac{1}{2\left(Z^{2}+x^{2}\right)} \frac{f\left(x^{2}\right) g\left(\frac{x}{\gamma}\right)-f\left(Z^{2}\right) g\left(\frac{Z}{\gamma}\right)-\frac{\partial}{\partial Z}\left(f\left(Z^{2}\right) g\left(\frac{Z}{\gamma}\right)\right)(Z-x)}{(Z-x)^{2}} d x \\
& \quad+\int_{|Z-x|>\epsilon} \frac{1}{2\left(Z^{2}+x^{2}\right)} \frac{f\left(x^{2}\right) g\left(\frac{x}{\gamma}\right)}{(Z-x)^{2}} d x \\
& \quad+\int_{|Z+x|<\epsilon} \frac{1}{2\left(Z^{2}+x^{2}\right)} \frac{f\left(x^{2}\right) g\left(\frac{x}{\gamma}\right)-f\left(Z^{2}\right) g\left(-\frac{Z}{\gamma}\right)-\frac{\partial}{\partial Z}\left(f\left(Z^{2}\right) g\left(-\frac{Z}{\gamma}\right)\right)(Z+x)}{(Z+x)^{2}} d x \\
& \quad+\int_{|Z+x|>\epsilon} \frac{1}{2\left(Z^{2}+x^{2}\right)} \frac{f\left(x^{2}\right) g\left(\frac{x}{\gamma}\right)}{(Z+x)^{2}} d x .
\end{aligned}
$$


Remark. The regularization is available because

$$
\int\left(\frac{\gamma}{\left(Z^{2}-\gamma^{2} Y^{2}\right)^{2}}\right)_{\mathrm{reg}} f\left(\gamma^{2} Y^{2}\right) g(Y) d Y=\int\left(\frac{1}{\left(Z^{2}-x^{2}\right)^{2}}\right)_{\mathrm{reg}} f\left(x^{2}\right) g\left(\frac{x}{\gamma}\right) d x .
$$

Proof. Using the identity

$$
\frac{\partial}{\partial Z}\left(f\left(Z^{2}\right) g\left(\frac{Z}{\gamma}\right)\right)=2 Z f^{\prime}\left(Z^{2}\right) g\left(\frac{Z}{\gamma}\right)+\frac{1}{\gamma} f\left(Z^{2}\right) g^{\prime}\left(\frac{Z}{\gamma}\right)
$$

where

$$
f^{\prime}(x)=\frac{\partial}{\partial x} f(x)
$$

and calculating the limit as $\gamma \rightarrow \infty$ of the right-hand side of (7), we get

$$
\begin{aligned}
& \lim _{\gamma \rightarrow \infty}\left(\left(\frac{\gamma}{\left(Z^{2}-\gamma^{2} Y^{2}\right)^{2}} f\left(\gamma^{2} Y^{2}\right)\right)_{\mathrm{reg}}, g\right) \\
& =\lim _{\gamma \rightarrow \infty}\left(\int_{|Z-x|<\epsilon} \frac{1}{2\left(Z^{2}+x^{2}\right)} \frac{f\left(x^{2}\right) g\left(\frac{x}{\gamma}\right)-f\left(Z^{2}\right) g\left(\frac{Z}{\gamma}\right)-\frac{\partial}{\partial Z}\left(f\left(Z^{2}\right) g\left(\frac{Z}{\gamma}\right)\right)(x-Z)}{(Z-x)^{2}} d x\right. \\
& \left.+\int_{|Z-x|>\epsilon} \frac{1}{2\left(Z^{2}+x^{2}\right)} \frac{f\left(x^{2}\right) g\left(\frac{x}{\gamma}\right)}{(Z-x)^{2}} d x\right) \\
& +\lim _{\gamma \rightarrow \infty}\left(\int_{|Z+x|<\epsilon} \frac{1}{2 Z} \frac{f\left(x^{2}\right) g\left(\frac{x}{\gamma}\right)-f\left(Z^{2}\right) g\left(-\frac{Z}{\gamma}\right)-\frac{\partial}{\partial Z}\left(f\left(Z^{2}\right) g\left(-\frac{Z}{\gamma}\right)\right)(Z+x)}{Z+x} d x\right. \\
& \left.+\int_{|Z+x|>\epsilon} \frac{1}{2\left(Z^{2}+x^{2}\right)} \frac{f\left(x^{2}\right) g\left(\frac{x}{\gamma}\right)}{(Z+x)^{2}} d x\right) \\
& =g(0)\left(\int_{|Z-x|<\epsilon} \frac{1}{2\left(Z^{2}+x^{2}\right)} \frac{f\left(x^{2}\right)-f\left(Z^{2}\right)-\left(\frac{\partial}{\partial Z} f\left(Z^{2}\right)\right)(Z-x)}{(Z-x)^{2}} d x\right. \\
& \left.+\int_{|Z-x|>\epsilon} \frac{1}{2\left(Z^{2}+x^{2}\right)} \frac{f\left(x^{2}\right)}{(Z-x)^{2}} d x\right) \\
& +g(0)\left(\int_{|Z+x|<\epsilon} \frac{1}{2\left(Z^{2}+x^{2}\right)} \frac{f\left(x^{2}\right)-f\left(Z^{2}\right)-\left(\frac{\partial}{\partial Z} f\left(Z^{2}\right)\right)(Z+x)}{(Z+x)^{2}} d x\right. \\
& \left.+\int_{|Z+x|>\epsilon} \frac{1}{2\left(Z^{2}+x^{2}\right)} \frac{f\left(x^{2}\right)}{(Z+x)^{2}} d x\right) \\
& =g(0)\left(\int \frac{f\left(x^{2}\right)}{2\left(Z^{2}+x^{2}\right)}\left(\frac{1}{(Z-x)^{2}}\right)_{\mathrm{reg}} d x+\int \frac{f\left(x^{2}\right)}{2\left(Z^{2}+x^{2}\right)}\left(\frac{1}{(Z+x)^{2}}\right)_{\mathrm{reg}} d x\right) \\
& \equiv g(0) \int f\left(x^{2}\right)\left(\frac{1}{\left(Z^{2}-x^{2}\right)^{2}}\right)_{\text {reg }} d x \text {. }
\end{aligned}
$$

We have used the fact that the coefficient of $g(Z / \gamma)$ tends to zero as $\gamma \rightarrow \infty$.

\section{$\S 3$. SHOCK WAVE IN dS}

Now we apply the above regularization procedure to get a shock wave that is an ultrarelativistic limit of the $D$-dimensional Schwarzschild-de-Sitter solution (here we consider the cases of $D=4$ and $D=5$ ). We also show that the result does not depend on the choice of the smoothing parameter $\epsilon$ and can be obtained by subtraction.

Formally, this shock wave was obtained in 2, without mathematical justification. For this reason, we do not write the initial metric and present only the integral representation 
that requires regularization. Note that in the formula below the parameter $a$ is related to the cosmological radius of the $\mathrm{dS}$ space-time, and $Z$ is one of the coordinates.

The integral defining the metric limit looks like this:

$$
\int_{-\infty}^{\infty} \frac{\left(Z^{2}\left(a^{2}-Z^{2}\right)+\left(a^{2}+Z^{2}\right) x^{2}\right)}{\left(Z^{2}-x^{2}\right)^{2}\left(a^{2}+x^{2}-Z^{2}\right)^{\frac{3}{2}}} d x .
$$

We see that this integral has two second order poles on the real axis at the points $x= \pm Z$. In accordance with the above regularization procedure and Lemma 3 , we have

$$
P_{\text {reg }}(Z, \epsilon)=P_{\text {reg }}^{(1)}+P_{\text {reg }}^{(2)}
$$

where

$$
\begin{aligned}
P_{\mathrm{reg}}^{(1)}= & \int_{|Z-x|<\epsilon} \frac{a^{2}+Z^{2}}{2\left(Z^{2}+x^{2}\right)} \frac{1}{\left(a^{2}+x^{2}-Z^{2}\right)^{\frac{3}{2}}} d x \\
& +\int_{|Z-x|>\epsilon} \frac{\left[Z^{2}\left(a^{2}-Z^{2}\right)+\left(a^{2}+Z^{2}\right) x^{2}\right]}{(Z+x)^{2}(Z-x)^{2}} \frac{1}{\left(a^{2}+x^{2}-Z^{2}\right)^{\frac{3}{2}}} d x, \\
P_{\mathrm{reg}}^{(2)}= & \int_{|Z+x|<\epsilon} \frac{a^{2}+Z^{2}}{2\left(Z^{2}+x^{2}\right)} \frac{1}{\left(a^{2}+x^{2}-Z^{2}\right)^{\frac{3}{2}}} d x \\
& +\int_{|Z+x|>\epsilon} \frac{\left[Z^{2}\left(a^{2}-Z^{2}\right)+\left(a^{2}+Z^{2}\right) x^{2}\right]}{(Z-x)^{2}(Z+x)^{2}} \frac{1}{\left(a^{2}+x^{2}-Z^{2}\right)^{\frac{3}{2}}} d x .
\end{aligned}
$$

First, we consider $P_{\text {reg. As }}^{(1)} \rightarrow 0$, the first term on the right in (9) is small and the asymptotics of the second term is given by the sum of two terms related to the boundary points:

$$
\operatorname{Sing} \operatorname{Part}\left(P_{\text {reg }}^{(1)}\right)=\left.\operatorname{Sing} \operatorname{Part}\left(P_{\text {reg }}^{(1)}\right)\right|_{Z-x \sim \epsilon}+\left.\operatorname{Sing} \operatorname{Part}\left(P_{\text {reg }}^{(1)}\right)\right|_{Z-x \sim-\epsilon},
$$

where

$$
\begin{aligned}
\left.\operatorname{Sing} \operatorname{Part}\left(P_{\text {reg }}^{(1)}\right)\right|_{Z-x \sim \epsilon} & \sim \frac{1}{\left(a^{2}\right)^{\frac{3}{2}}} \int^{Z-\epsilon} \frac{\left[Z^{2}\left(a^{2}-Z^{2}\right)+\left(a^{2}+Z^{2}\right) Z^{2}\right]}{4 Z^{2}(Z-x)^{2}} d x, \\
\text { Sing Part_ }\left.P_{\text {reg }}^{(1)}\right|_{Z-x \sim-\epsilon} & \sim \frac{1}{\left(a^{2}\right)^{\frac{3}{2}}} \int_{Z+\epsilon} \frac{\left[Z^{2}\left(a^{2}-Z^{2}\right)+\left(a^{2}+Z^{2}\right) Z^{2}\right]}{4 Z^{2}(Z-x)^{2}} d x .
\end{aligned}
$$

Calculating integrals (11) and (12) explicitly, we get

$$
\begin{aligned}
\left.\operatorname{Sing} \operatorname{Part}\left(P_{\text {reg }}^{(1)}\right)\right|_{Z-x \sim \epsilon} & =(Z-x=y) \\
& =\frac{1}{\left(a^{2}\right)^{\frac{3}{2}}} \int^{\epsilon} \frac{\left[Z^{2}\left(a^{2}-Z^{2}\right)+\left(a^{2}+Z^{2}\right) Z^{2}\right]}{4 Z^{2} y^{2}} d y \\
& =\frac{1}{\left(a^{2}\right)^{\frac{3}{2}}} \int_{\epsilon} \frac{a^{2}}{2 y^{2}} d y=\frac{1}{2 a} \int_{\epsilon} \frac{1}{y^{2}} d y \sim \frac{1}{2 a} \frac{1}{\epsilon}, \\
\left.\operatorname{Sing} \operatorname{Part} P_{\text {reg }}^{(1)}\right|_{Z-x \sim-\epsilon} & =(Z-x=-y \rightarrow y=x-Z) \\
& =\frac{1}{\left(a^{2}\right)^{\frac{3}{2}}} \int_{\epsilon} \frac{a^{2}}{2 y^{2}} d x=\frac{1}{2 a} \int_{\epsilon} \frac{1}{y^{2}} d x \sim \frac{1}{2 a} \frac{1}{\epsilon} .
\end{aligned}
$$

Therefore, as expected, we have a singularity as $\epsilon \rightarrow 0$. But it does not depend on $Z$, which allows us to subtract it from the expression without loss of the physical meaning. 
Indeed,

$$
\begin{aligned}
& P_{\text {reg }}(Z, \epsilon)=P_{\text {reg }}^{(1)}+P_{\text {reg }}^{(2)} \\
& =\int_{|Z-x|<\epsilon} \frac{a^{2}+Z^{2}}{2\left(Z^{2}+x^{2}\right)} \frac{1}{\left(a^{2}+x^{2}-Z^{2}\right)^{\frac{3}{2}}} d x \\
& +\int_{|Z-x|>\epsilon} \frac{\left[Z^{2}\left(a^{2}-Z^{2}\right)+\left(a^{2}+Z^{2}\right) x^{2}\right]}{(Z+x)^{2}(Z-x)^{2}} \frac{1}{\left(a^{2}+x^{2}-Z^{2}\right)^{\frac{3}{2}}} d x \\
& +\int_{|Z+x|<\epsilon} \frac{a^{2}+Z^{2}}{2\left(Z^{2}+x^{2}\right)} \frac{1}{\left(a^{2}+x^{2}-Z^{2}\right)^{\frac{3}{2}}} d x \\
& +\int_{|Z+x|>\epsilon} \frac{\left[Z^{2}\left(a^{2}-Z^{2}\right)+\left(a^{2}+Z^{2}\right) x^{2}\right]}{(Z-x)^{2}(Z+x)^{2}} \frac{1}{\left(a^{2}+x^{2}-Z^{2}\right)^{\frac{3}{2}}} d x \\
& =\int_{-Z+\epsilon}^{-Z-\epsilon} \frac{a^{2}+Z^{2}}{2\left(Z^{2}+x^{2}\right)} \frac{1}{\left(a^{2}+x^{2}-Z^{2}\right)^{\frac{3}{2}}} d x \\
& +\int_{Z+\epsilon}^{Z-\epsilon} \frac{a^{2}+Z^{2}}{2\left(Z^{2}+x^{2}\right)} \frac{1}{\left(a^{2}+x^{2}-Z^{2}\right)^{\frac{3}{2}}} d x \\
& +\left(\int_{-\infty}^{-Z-\epsilon}+\int_{-Z+\epsilon}^{Z-\epsilon}+\int_{Z+\epsilon}^{\infty}\right) \frac{\left[Z^{2}\left(a^{2}-Z^{2}\right)+\left(a^{2}+Z^{2}\right) x^{2}\right]}{\left(Z^{2}-x^{2}\right)^{2}} \\
& \times \frac{1}{\left(a^{2}+x^{2}-Z^{2}\right)^{\frac{3}{2}}} d x \\
& =I_{4}(Z)+\left.\operatorname{Sing} \operatorname{Part} P_{\text {reg }}^{(1)}\right|_{Z-x \sim-\epsilon}+\left.\operatorname{Sing} \operatorname{Part} P_{\text {reg }}^{(1)}\right|_{Z-x \sim \epsilon} \\
& + \text { Sing Part }\left.P_{\text {reg }}^{(2)}\right|_{Z+x \sim-\epsilon}+\operatorname{Sing} \text { Part }\left.P_{\text {reg }}^{(2)}\right|_{Z+x \sim \epsilon}+\mathcal{O}(\epsilon) \\
& =I_{4}(Z)+\frac{1}{2 a} \frac{2}{\epsilon}+\frac{1}{2 a} \frac{2}{\epsilon}+\mathcal{O}(\epsilon) \text {. }
\end{aligned}
$$

In other words, the integral (8) can be understood in the principal value sense near the points $x=Z$ and $x=-Z$. The independence of the singular term of $Z$ makes it possible to perform renormalization simply by removing this term.

Therefore, we have shown that the passage to the ultrarelativistic limit can be performed not only for the case where the components of the initial metric are integrable functions, but also in the cases where they are integrable in the sense of principal values:

$$
\text { p.v. } \int_{-\infty}^{\infty} \frac{\left(Z^{2}\left(a^{2}-Z^{2}\right)+\left(a^{2}+Z^{2}\right) x^{2}\right)}{\left(Z^{2}-x^{2}\right)^{2}\left(a^{2}+x^{2}-Z^{2}\right)^{\frac{3}{2}}} d x=\frac{8}{a^{2}}\left[-1+\frac{Z_{4}}{2 a} \ln \left(\frac{a+Z_{4}}{a-Z_{4}}\right)\right]
$$

This confirms the mathematical correctness of a series of papers devoted to shock waves in the dS space-time [2]-9]. Finally, we consider the case where $D=5$. Then the integral to be regularized has the form

$$
\int \frac{\left(Z^{2}\left(a^{2}-Z^{2}\right)+\left(a^{2}+Z^{2}\right) x^{2}\right)}{\left(Z^{2}-x^{2}\right)^{2}\left(a^{2}+x^{2}-Z^{2}\right)^{2}} d x=\frac{3 \pi}{2 a^{3}} \frac{-1+\frac{2 Z^{2}}{a^{2}}}{\sqrt{1-\frac{Z^{2}}{a^{2}}}}
$$


Omitting explicit calculations, we only present the singular term near the point $x=Z$ :

$$
\begin{aligned}
\left(\int_{Z-\epsilon}^{Z+\epsilon}+\int_{-Z-\epsilon}^{-Z+\epsilon}\right) \frac{\left[Z^{2}\left(a^{2}-Z^{2}\right)+\left(a^{2}+Z^{2}\right) x^{2}\right]}{(Z-x)^{2}(Z+x)^{2}} \frac{1}{\left(a^{2}+x^{2}-Z^{2}\right)^{2}} d x \\
=\frac{\left[Z^{2}\left(a^{2}-Z^{2}\right)+\left(a^{2}+Z^{2}\right) Z^{2}\right]}{4 Z^{2}\left(a^{2}\right)^{2}}\left(\int_{Z-\epsilon}^{Z+\epsilon}\right) \frac{1}{(Z-x)^{2}} d z \\
\quad+\frac{\left[Z^{2}\left(a^{2}-Z^{2}\right)+\left(a^{2}+Z^{2}\right) Z^{2}\right]}{4 Z^{2}\left(a^{4}\right)}\left(\int_{-Z-\epsilon}^{-Z+\epsilon}\right) \frac{1}{(Z+x)^{2}} d x \\
=\frac{2 Z^{2} a^{2}}{4 Z^{2}\left(a^{2}\right)^{4}}\left(\int_{Z-\epsilon}^{Z+\epsilon} \frac{1}{(Z-x)^{2}} d x+\int_{-Z-\epsilon}^{-Z+\epsilon} \frac{1}{(Z+x)^{2}} d x\right) \\
=\frac{1}{2 a^{2}}\left(-\frac{4}{\epsilon}\right) .
\end{aligned}
$$

As before, this singular term does not depend on $Z$ and can be subtracted.

\section{REFERENCES}

[1] P. Aichelburg and R. Sexl, On the gravitational field of a massless particle, Gen. Relativity Gravitation 2 (1971), 303.

[2] M. Hotta and M. Tanaka, Shock wave geometry with nonvanishing cosmological constant, Classical Quantum Gravity 10 (1993), 307-314. MR.1205215 (94b:83022)

[3] J. B. Griffiths, Colliding plane waves in general relativity, Clarendon Press, New York, 1991. MR $1135109(92 \mathrm{k}: 83036)$

[4] J. Podolský and J. B. Griffiths, Nonexpanding impulsive gravitational waves with an arbitrary cosmological constant, Phys. Lett. A 261 (1999), 1-4; arXiv:gr-qc/9908008. MR1718186 (2000f:83024)

[5] J. Podolský, Exact impulsive gravitational waves in spacetimes of constant curvature, arXiv: gr-qc/0201029.

[6] J. Podolský and M. Ortaggio, Symmetries and geodesics in (anti-) de Sitter space-times with nonexpanding impulsive waves, Classical Quantum Gravity 18 (2001), 2689-2706; arXiv: gr-qc/0105065. MR $1846367(2002 \mathrm{~h}: 83029)$

[7] K. Sfetsos, On gravitational shock waves in curved space-times, Nuclear Phys. B 436 (1995), 721745; arXiv:hep-th/9408169. MR1316141 (95k:83067)

[8] I. Ya. Aref'eva, A. A. Bagrov, and E. A. Guseva, Critical formation of trapped surfaces in the collision of non-expanding gravitational shock waves in de Sitter space-time, arXiv:0905.1087. MR2593027 (2011a:83029)

[9] I. Ya. Aref'eva, A. A. Bagrov, and L. V. Joukovskaya, Critical trapped surfaces formation in the collision of ultrarelativistic charges in $(A) d S$, arXiv:0909.1294.

[10] N. Arkani-Hamed, S. Dimopoulos, and G. R. Dvali, The hierarchy problem and new dimensions at a millimeter, Phys. Lett. B 429 (1998), 263; arXiv: hep-ph/9803315.

[11] I. Antoniadis, N. Arkani-Hamed, S. Dimopoulos, and G. R. Dvali, New dimensions at a millimeter to a Fermi and superstrings at a TeV, Phys. Lett. B 436 (1998), 257; arXiv: hep-ph/9804398.

[12] G. F. Giudice, R. Rattazzi, and J. D. Wells, Quantum gravity and extra dimensions at high-energy colliders, Nuclear Phys. B 544 (1999), 3; arXiv:hep-ph/9811291. MR/1809052 (2001k:81364)

[13] _ Transplanckian collisions at the LHC and beyond, Nuclear Phys. B 630 (2002), 293; arXiv:hep-ph/0112161. MR/1809052 (2001k:81364)

[14] T. Banks and W. Fischler, A model for high energy scattering in quantum gravity, arXiv: hep-th/9906038.

[15] I. Ya. Aref'eva, High-energy scattering in the brane world and black hole production, Part. Nuclear 31 (2000), 169; arXiv:hep-th/9910269.

[16] S. Dimopoulos and G. Landsberg, Black holes at the LHC, Phys. Rev. Lett. 87 (2001), 161602; arXiv: hep-ph/0106295.

[17] S. B. Giddings and S. Thomas, High energy colliders as black hole factories: The end of short distance physics, Phys. Rev. D 65 (2002), 056010; arXiv:hep-ph/0106219. MR2497645

[18] G. 't Hooft, Graviton dominance in ultrahigh-energy scattering, Phys. Lett. B 198 (1987), 61.

[19] _ On the factorization of universal poles in a theory of gravitating point particles, Nuclear Phys. B 304 (1988), 867-876. MR0952774 (89j:83039) 
[20] T. Dray and G. 't Hooft, The gravitational shock wave of a massless particle, Nuclear Phys. B 253 (1985), 173-188. MR0789737(86f:83021)

[21] P. D. D'Eath and P. N. Payne, Gravitational radiation in black-hole collisions at the speed of light. I. Perturbation treatment of the axisymmetric collision, Phys. Rev. D 46 (1992), 658-674. MR 1172255 (93j:83053)

[22] - Gravitational radiation in black-hole collisions at the speed of light. II. Reduction to two independent variables and calculation of the second-order news function, Phys. Rev. D 46 (1992), 675-693. MR 1172256 (93j:83054)

[23] _ Gravitational radiation in black-hole collisions at the speed of light. III. Results and conclusions, Phys. Rev. D 46 (1992), 694-701. MR.1172257 (93j:83055)

[24] R. Steinbauer and J. A. Vickers, The use of generalized functions and distributions in general relativity, Classical Quantum Gravity 23 (2006), R91-R114; 2006; arXiv:gr-qc/0603078. MR2226026 (2007d:83018)

[25] H. Balasin, Distributional energy-momentum tensor of the extended Kerr geometry, Classical Quantum Gravity 14 (1997), 3353-3362; arXiv: gr-qc/9702060. MR.1492277 (99f:83009)

[26] H. Balasin and H. Nachbagauer, On the distributional nature of the energy-momentum tensor of a black hole or What curves the Schwarzschild geometry? Classical Quantum Gravity 10 (1993), 2271-2278; arXiv:gr-qc/9305009. MR.1243970(94k:83044)

[27] H. Balasin, Geodesics for impulsive gravitational waves and the multiplication of distributions, Classical Quantum Gravity 14 (1997), 455-462; arXiv:gr-qc/9607076. MR.1437438 (98a:83046)

[28] I. M. Gel'fand and G. E. Shilov, Generalized functions. Vols. 1, 2, Fizmatgiz, Moscow, 1958; English transl., Acad Press, New York-London, 1964; 1968. MR0097715 (20:4182) MR0106409 (21:5142a) MR0435831(55:8786a) MR0435832(55:8786b)

[29] V. S. Vladimirov, Generalized functions in mathematical physics, Nauka, Moscow, 1976; English transl., Mir, Moscow, 1979. MR0450966 (56:9256) MR0564116 (80j:46062b)

V. A. Steklov Mathematical Institute, Gubkina 42, Moscow 191011, Russia

E-mail address: arefeva@mi.ras.ru

V. A. Steklov Mathematical Institute, Gubkina 42, Moscow 191011, Russia

E-mail address: andrey@googlemail.com

Centre for Theoretical Cosmology, Damtp, CMS, University of Cambridge, Wilberforce Road, CB3 0WA, Cambridge, United Kingdom

Received 26/JAN/2010

Translated by THE AUTHORS 